\title{
Residual Centering, Exploratory and Confirmatory Moderator Analysis, and Decomposition of Effects in Path Models Containing Interactions
}

\author{
Charles E. Lames \\ University of Georgim
}

\begin{abstract}
Hierarchical moderated regression (HMR) analysis may lead to interpretational problems in tests of moderator (interaction) hypotheses. An alternative, residual-centering approach is described and compared to traditional HMR analysis. Procedures for evaluating interaction hypotheses and general effect analysis proce-
\end{abstract}

\begin{abstract}
dures are described for path (causal) models containing interactions. Index terms: Confirmatory analysis, Effect analysis in path models, Goodness-offit tests, Hierarchical moderated regression, Mediators, Moderated regression, Multicollinearity, Path models, Residual centering.
\end{abstract}

Hierarchical moderated regression (HMR) analysis yields appropriate tests of interaction hypotheses in experimental and nonexperimental data (Arnold, 1982, 1984; Cohen, 1978; Cohen \& Cohen, 1975; Saunders, 1956; Zedeck, 1971). However, various problems are often encountered with HMR analysis in field research, including (1) multicollinearity due to high correlation between cross-product and main effect terms, (2) inflated standard errors and unstable regression estimates, (3) unpredictable suppressor or "enhancer" effects (McFatter, 1979), and (4) uninterpretable regression parameter estimates. Various centering methods (e.g., Tate, 1984) and biased regression procedures (e.g., Bentler \& Woodward, 1979; Morris, Sherman, \& Mansfield, 1986) have been proposed as analytic alternatives to overcome these limitations to HMR analysis. The purposes of this paper are (1) to describe an alternative, cross-product residual-centering approach to regression tests of interaction effects, (2) to illustrate this approach and compare it to HMR analysis, (3) to outline interaction hypothesis testing procedures in path models, and (4) to describe effect analysis procedures for path models containing interactions.

\section{Residilal Centering}

HMR analysis involves a comparison of $R^{2} \mathrm{~s}$ between one regression model that contains only linear terms, such as

$Y=b_{1} X_{1}+b_{2} X_{2}+d$,

and a second model that contains linear terms and a cross-product between predictors:

$Y=b_{1} X_{1}+b_{2} X_{2}+b_{3} X_{1 \times 2}+d$.

APPLIED PSYCHOLOGICAL MEASUREMENT

Vol. 12, No. 2, June 1988, pp.163-175

(C) Copyright 1988 Applied Psychological Measurement Inc.

$0146-6216 / 88 / 020163-13 \$ 1.90$ 
For algebraic convenience, all terms in Equations 1 and 2 are expressed in standard score form, $b_{1}$ and $b_{2}$ are the ordinary least squares (OLS) weights from the regression of $Y$ on $X_{1}$ and $X_{2}, d s$ are oLs residuals, and in Equation $2 b_{3}$ is the oLs regression weight associated with the $X_{1} \times X_{2}$ cross-product term $X_{1 \times 2}$ (Arnold, 1982, 1984; Cohen \& Cohen, 1975). An interaction hypothesis is supported if the $\mathbb{R}^{2}$ from the Equation 2 regression model is significantly larger than the Equation $\mathbb{1} \mathbb{R}^{2}$ (Cohen, 1978). This simultaneously tests for homogeneity of slope of the regression of the dependent variable on one predictor across all levels of the second predictor (Amold, 1982; Gulliksen \& Wilks, 1950).

In field research, the $X_{1 \times 2}$ cross-product term in Equation 2 will often be highly correlated with $X_{1}$ and/or $X_{2}$, leading to interpretational problems associated with multicollinearity (Belsley, Kuh, \& Welsch, 1980). Furthermore, the $X_{1 \times 2}$ term in Equation 2 confounds the interaction and predictor main effects (Stone \& Hollenbeck, 1984). An alternative approach that avoids these problems begins with a regression of the cross-product term on main effects, for example:

$X_{1 \times 2}=c_{1} X_{1}+c_{2} X_{2}+d$.

In the second step, cross-product residuals are constructed:

$d_{1 \times 2}=X_{1 \times 2}-\hat{X}_{1 \times 2}=X_{1 \times 2}-\left(\hat{c}_{1} X_{1}+\hat{c}_{2} X_{2}\right)$.

$d_{1 \times 2}$ may be restandardized, and then used in the full equation regression:

$Y=b_{1} X_{1}+b_{2} X_{2}+b_{3} d_{1 \times 2}+d_{Y}$.

The decision to rescale $d_{1 \times 2}$ should be based, in part, on whether the original variables were measured on some non-arbitrary metric (Stolzenberg, 1980). If they were, unstandardized regression estimates should be used in Equations 4 and 5 to preserve the metrics of the original variables. However, for many psychological variables that have arbitrary scales, standardization may aid interpretation by placing all variables on a common measurement scale. ${ }^{1}$

The difference $R^{2}$ comparing the models in Equations 1 and $5\left(\Delta R_{5-1}^{2}=R_{5}^{2}-R_{1}^{2}\right)$ will equal the difference $R^{2}$ between the models in Equations 1 and $2\left(\Delta R_{2-1}^{2}=\mathbb{R}_{2}^{2}-R_{1}^{2}\right)$, but the $\Delta R_{5-1}^{2} F$-test (Kerlinger \& Pedhazur, 1973) will have degrees of freedom equal to 1 and $n-p-3$ with $n$ observations and $p$ predictors (compared with degrees of freedom of 1 and $n-p-1$ for $\Delta R_{2-1}^{2}$ ). Thus the $\Delta R_{5-1}^{2}$ test will be slightly less powerful (negligibly so with large samples) but has a number of advantages: It results in reduced multicollinearity among predictors as well as smaller standard errors, it separates interaction and main effects, and (with standardized data) it yields a regression coefficient for the residualized crossproduct term that is directly interpretable as the effect of the $X_{1} \times X_{2}$ interaction on $Y$. As in traditional HMR analysis, a finding of a significant interaction effect is appropriately followed by subgroup regression analyses of the form of the interaction (Arnold, 1982).

\section{An Example}

As part of another study, it was hypothesized that rater cognitive complexity would have stronger effects on memory for ratee behaviors under high (rather than low) memory demand conditions. Undergraduate psychology students $(N=207)$ at the University of Georgia completed Vannoy's (1965) version of Bieri's Role Construct Repertory Grid measure of Cognitive Complexity. They then read one of two

\footnotetext{
'A reviewer also pointed out that standard errors of standardized regression weights for stochastic predictors are miscalculated by many regression packages, although these effects may often be minor. Bentler and Lee (1983, pp. 212-214) discussed this problem and proposed a solution using polynomial constraints in the analysis of covariance structures. Correct statistical inferences can also be drawn from standard errors of corresponding unstandardized weights.
} 
randomly assigned scripts (differing in overall favorability) of a hypothetical college lecture. Respondents were randomly assigned to one of two Memory Demand conditions; one group completed a recognition test of memory for behavioral incidents immediately after reading a script, and the other group was tested two days later. (Memory Demand was coded $1=$ immediate rating, $2=$ two-day delay.) The memory test included equal numbers of behavioral incidents that were or were not included in the script read previously. The dependent variable, memory for behaviors, was measured as the number of incidents correctly identified as having been included, plus the number of incidents correctly identified as having not been included in the script.

Correlations among variables are shown in Table 1. Note that (1) the Memory Demand $\times$ Cognitive Complexity cross-product $\left(X_{1 \times 2}\right)$ was highly correlated with main effects, creating the potential for multicollinearity in HMR analysis; (2) residual centering effectively eliminated collinearity; (3) the Memory Demand $\times$ Cognitive Complexity residual $\left(d_{1 \times 2}\right)$ is empirically distinguishable from the cross-product term; and (4) the residual (interaction) term is significantly correlated with the dependent variable, while the cross-product term is not.

Table 2 compares results from a traditional HMR analysis and the present approach in testing for interaction effects between Memory Demand $\left(X_{1}\right)$ and Cognitive Complexiry $\left(X_{2}\right)$ on Memory for Behaviors $(Y)$. Models I, II, and III correspond to regression models in Equations 1, 2, and 5, respectively. For ease of comparison, standardized regression parameter estimates (bs) and standard errors (SES) are shown.

Comparisons between Models $\mathbb{I}$ and $\mathbb{I}$ suggest the presence of multicollinearity among predictors: Coefficients and standard errors are inflated in Model III estimates. The Memory Demand $\times$ Cognitive Complexity cross-product term also served as a suppressor or enhancer variable (McFatter, 1979) in the Model II equation: The $X_{1}$ and $X_{2}$ regression weights are much larger than corresponding zero-order correlations with the criterion (Tzelgov \& Henik, 1985). Thus Model II parameter estimates themselves are not easily interpreted. However, the difference $R^{2}$ comparing Models $I$ and $I I$ is significant $(F=$ $5.65, p<.05)$, and suggests the presence of a significant interaction effect.

On the other hand, Model III regression estimates have smaller standard errors than their Model II counterparts, and are more interpretable. In particular, the statistically significant $d_{1 \times 2}$ coefficient may be interpreted as (1) the standard unit change in $Y$ per standard unit change in the Memory Demand $X$ Cognitive Complexity interaction (Stolzenberg, 1980), and (2) the square root of the incremental proportion of variance in $Y$ explained by the interaction effect (Cohen, 1978).

Table 3 compares HMR and residual-centering analyses in terms of several collinearity diagnostics: Belsley et al.'s (1980) singular-value decomposition (SVD) of the $n \times p$ predictor data matrix $\mathbb{X}$, and Chatterjee and Price's (1977) variance inflation factors (VIFs). Chatterjee and Price defined $\mathrm{VIF}_{i}=$

Table 1

Correlations Among Variables

for the Memory For Ratee Behaviors Experiment

\begin{tabular}{llllll}
\hline Variable & $\mathrm{Y}$ & $\mathrm{X}_{1}$ & $\mathrm{X}_{2}$ & $\mathrm{X}_{1 \times 2}$ & $\mathrm{~d}_{1 \times 2}$ \\
\hline Memory for Behavioxs $(Y)$ & 1.000 & & & & \\
Memory Demand $\left(\mathrm{X}_{1}\right)$ & & -.112 & 1.000 & & \\
Cognitive Complexity $\left(\mathrm{X}_{2}\right)$ & -.017 & $-.176 *$ & 1.000 & & \\
$\mathrm{X}_{1} \times \mathrm{X}_{2}$ Cross-product $\left(\mathrm{X}_{1 \times 2}\right)$ & -.073 & $.756 * *$ & $.475 * *$ & 1.000 & \\
$\mathrm{X}_{1} \times \mathrm{X}_{2}$ Residual $\left(\mathrm{d}_{1 \times 2}\right)$ & $.163 *$ & .000 & .000 & $.217 * * 1.000$ \\
\hline
\end{tabular}

$x_{\mathrm{p}}<.05 ; * * \mathrm{x}<.01$ 
Table 2

Standaxdized Regression Analyses of the Cognitive Complexity $\times$ Memory Demand Effect on Memory For Behaviors

\begin{tabular}{|c|c|c|c|c|c|}
\hline Predictors & $x_{1}$ & $\mathrm{x}_{2}$ & $x_{1 \times 2}$ & $\mathrm{~d}_{1 \times 2}$ & $\mathrm{R}^{2}$ \\
\hline \multicolumn{6}{|l|}{ HMR Analysis } \\
\hline \multicolumn{6}{|l|}{ Model I (Equation 1) } \\
\hline $\mathrm{b}$ & -.119 & -.038 & $\therefore$ & $\ldots$ & .014 \\
\hline $\mathrm{SE}$ & .070 & .070 & & & \\
\hline \multicolumn{6}{|l|}{ Model II (Equation 2) } \\
\hline b & $-.773 x+2 x$ & $-.512 * \%$ & $.755 * x$ & $\ldots$ & $.041 *$ \\
\hline $\mathrm{SE}$ & 284 & .211 & .317 & & \\
\hline \multirow{2}{*}{\multicolumn{6}{|c|}{$\begin{array}{c}\text { Residual Centering Analysis } \\
\text { Model III (Equation 5) }\end{array}$}} \\
\hline & & & & & \\
\hline $\mathrm{b}$ & -.119 & -.038 & $\cdots$ & $.163 *$ & $.041 *$ \\
\hline $\mathrm{SE}$ & .070 & .070 & & .069 & \\
\hline
\end{tabular}

$* p<.05 ; * * p<.01$

$1 /\left(1-R_{i}^{2}\right)$, where $R_{i}^{2}$ is the squared multiple correlation from the regression of the $i$ th predictor on the $p-1$ other predictors. Belsley et al. (1980) defined the $k$ th condition index as $\lambda_{k}=\left(\lambda_{\max }\right)^{1 / 2} /\left(\lambda_{k}\right)^{1 / 2}$, where $\lambda_{\max }$ and $\lambda_{k}$ refer to the maximum and $k$ th eigenvalues of $\mathbf{X}^{\prime} \mathbb{X}$, respectively. The $k$ th singular value is $\left(\lambda_{k}\right)^{1 / 2}$. The condition number of the $X$ matrix is given as $k(X)=\left(\lambda_{\max }\right)^{1 / 2} /\left(\lambda_{\min }\right)^{1 / 2}$, where $\lambda_{\min }$ refers to the minimum eigenvalue of $\mathbb{X}^{\prime} \mathbf{X}$. The $i, k$ th variance-decomposition proportion represents the proportion of the variance of the ith regression coefficient associated with the $k$ th component of its decomposition (Belsley et al., 1980, p. 106).

The presence of multicollinearity is signaled by (1) one or more near-zero eigenvalues (Morris et al., 1986), (2) one or more "high" condition indices", (3) "high" VIFs (e.g., VIF $>$ 10, Johnston, 1984), and (4) two or more large variance-decomposition proportions associated with a high condition index (see Belsley, 1984; Belsley et al., 1980). For HMR analysis Table 3 shows (1) one small eigenvalue $(.021),(2)$ one large condition index (9.274), (3) high VIFs (16.985 and 21.778), and (4) one row of large variance-decomposition proportions associated with the large condition index. These results are consistent in suggesting that for this dataset, HMR analysis is more likely to suffer ill effects of multicollinearity than residual-centering analysis.

In summary, residual-centering analysis of interaction hypotheses not only minimizes multicollinearity problems that may threaten HMR analysis, but also provides a straightforward means to assess the predictability of some criterion from the interaction among predictors.

Results in Table 2 supported a significant interaction effect between Memory Demand and Cognitive Complexity in predicting Memory for Behaviors. Follow-up subgroup standardized regression analysis showed that Cognitive Complexity $\left(X_{2}\right)$ had a significant effect on Memory for Behaviors $(Y)$ in the high Memory Demand condition $\left(\hat{Y}=.224 X_{2} ; p<.05\right)$ but not in the low Memory Demand condition $(\hat{Y}=$ $.150 \mathrm{X}_{2} ; p>10$ ). Expected values for $Y$, in its original metric, may also be easily calculated from unstandardized regression coefficients:

$Y=22.762-1.280 X_{1}-.008 X_{2}+.067 d_{1 \times 2}$,

"Belsley et al. (1980) referred to a condition index of 30 or above as "high" but also cautioned that condition numbers as low as 8 may signal ill-conditioned data. 
Table 3

Collinearity Diagnostics

\begin{tabular}{|c|c|c|c|c|}
\hline \multirow[b]{2}{*}{ Eigenvalue } & \multirow{2}{*}{$\begin{array}{l}\text { Condition } \\
\text { Index }\end{array}$} & \multicolumn{3}{|c|}{$\begin{array}{c}\text { Variance-Decomposition } \\
\text { Proportions }\end{array}$} \\
\hline & & $\mathrm{X}_{1}$ & $\mathrm{x}_{2}$ & $x_{1 \times 2}$ \\
\hline \multicolumn{5}{|l|}{ HMR Analysis } \\
\hline 1.837 & 1.000 & .012 & .006 & .013 \\
\hline 1.142 & 1.268 & .015 & .066 & .000 \\
\hline .021 & 9.274 & .974 & .928 & .986 \\
\hline Variance & Inflation Factors & 16.985 & 9.478 & 21.778 \\
\hline \multicolumn{5}{|c|}{ Residual Centering Analysis } \\
\hline 1.156 & 1.000 & .422 & .422 & .000 \\
\hline 1.000 & 1.075 & .000 & .000 & 1.000 \\
\hline .844 & 1.171 & .578 & .578 & .000 \\
\hline Variance & Inflation Factors & 1.025 & 1.025 & 1.000 \\
\hline
\end{tabular}

which, when substituting the estimates for

$d_{1 \times 2}=X_{1 \times 2}-\left(-147.809+104.589 X_{1}+1.392 X_{2}\right)$,

reduces to the usual oLs estimates:

$Y=32.713-8.306 X_{1}-.101 X_{2}+.067 X_{1 \times 2}$.

Thus the reduced-form residual-centered estimates are identical to oLS estimates.

\section{Moderators in Confirmatory Analysi̊s}

The focus so far has been on exploratory moderator analysis, but moderator effects can also be easily incorporated and tested in causal (path) models. Figure 1 shows the path model for the interaction hypothesis above. The model proposes that an interaction between Memory Demand and Cognitive Complexity $\left(d_{1 \times 2}\right)$ has a direct effect (solid line) on Memory for Behaviors. Memory Demand and Cognitive Complexity are presumed to not have direct effects (broken lines) on Memory for Behaviors.

This model is appropriately evaluated by first constructing cross-product residuals $d_{1 \times 2}$ as in Equation 4. The interaction hypothesis is then tested directly by regressing Memory for Behaviors $(Y)$ on $d_{1 \times 2}$. A significant standardized regression parameter estimate $(b=.163, p=.019)$ supported the hypothesis in the present example.

Next, a disturbance term regression test (Lance, 1986) evaluates additional hypotheses that neither Memory Demand $\left(X_{1}\right)$ nor Cognitive Complexity $\left(X_{2}\right)$ has a direct effect on Memory for Behaviors:

$\tilde{d}_{Y}=f_{1} X_{1}+f_{2} X_{2}+e$,

where $\tilde{d}_{Y}=Y-\hat{Y}=Y-\left(\hat{b} d_{1 \times 2}\right)$ are residuals from the regression of $Y$ on the $d_{1 \times 2}$ interaction term, and $e$ contains oLs residuals from the regression of $\tilde{d}_{y}$ on $X_{1}$ and $X_{2}$. A significant $\hat{f}_{1}$ and/or $\hat{f}_{2}$ would indicate model misspecification(s): Direct rather than indirect effects from $X_{1}$ and/or $X_{2}$ would be implied. In the present example, neither $\hat{f}_{1}=-.121(p>.05)$ nor $\hat{f}_{2}=-.039(p>.05)$ were significant, suggesting that the model had been correctly specified. This implies that any Cognitive Complexity effect on Memory for Behaviors is contingent on level of Memory Demand.

"Partial moderation" models may be similarly evaluated. In Figure $2 \mathrm{a}$, for example, both $X_{1}$ and the interaction between $X_{1}$ and $X_{2}$ are presumed to have direct effects on $Y$, whereas the effect of $X_{2}$ on 


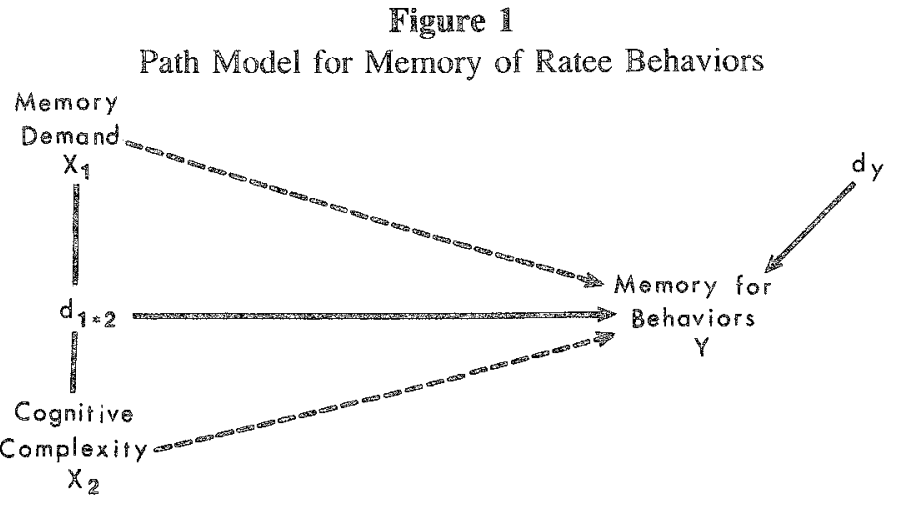

$Y$ is presumed to be indirect. This model is appropriately evaluated by creating $d_{1 \times 2}$ residuals and regressing $Y$ on $X_{1}$ and $d_{1 \times 2}$. Significant regression estimates would support model propositions. A nonsignificant $\hat{f}_{1}$ in

$\tilde{d}_{Y}=f_{1} X_{2}+e$,

where $\tilde{d}_{Y}=Y-\hat{Y}=Y-\left(\hat{b}_{1} X_{1}+\hat{b}_{2} d_{1 \times 2}\right)$, would support the additional hypothesis that $X_{2}$ has no direct effect on $Y$.

If the model in Figure $2 \mathrm{a}$ is supported empirically, direct effects of $X_{1}$ and the $X_{1} \times X_{2}$ interaction on $Y$ can be calculated by taking partial derivatives of the estimated structural equations (Stolzenberg, 1980):

$\hat{Y}=\hat{b}_{1} X_{1}+\hat{b}_{2} d_{1 \times 2}=\hat{b}_{1} X_{1}+\hat{b}_{2}\left(X_{1 \times 2}-\hat{c}_{1} X_{1}-\hat{c}_{2} X_{2}\right)$

with respect to explanatory variables:

$\partial Y / \partial X_{1}=\hat{b}_{1}+\hat{b}_{2}\left(X_{2}-\hat{c}_{1}\right)$

$\partial Y / \partial d_{1 \times 2}=\hat{b}_{2}$.

Although $X_{2}$ is not an explanatory variable in Figure 2a, it too has an effect on $Y$ that is confounded with the direct interaction effect:

$\partial Y / \partial X_{2}=\hat{b}_{2}\left(X_{1}-\hat{c}_{2}\right)$.

Equation 12 illustrates the intuitive motion that the direct main effect of $X_{1}$ on $Y$ cannot be interpreted independently of the direct $X_{1} \times X_{2}$ interaction effect: The direct main effect of $X_{1}$ must be evaluated separately at various levels of $X_{2}$. Equation $\mathbb{1} 3$ indicates a constant rate of change in $Y$ as a function of the interaction effect. Equation 14 shows that the effect of $X_{2}$ on $Y$ is linked solely to the interaction effect and must also be evaluated at various levels of $X_{1}$.

\section{Medingtors and Moderators}

A moderator is defined here as some measured variable, across whose values the relationship between two (or more) other variables varies. A mediator is defined as some measured variable that serves, in whole or in part, to transmit the causal effect of some antecedent variable on a consequent variable (James, Mulaik, \& Brett, 1982). Mediation (but not moderation) connotes causality, though causal relations may be moderated (James \& Brett, 1984). 
Figure 2

Hypothetical Path Models Containing Interactions

(a) Partial Moderation

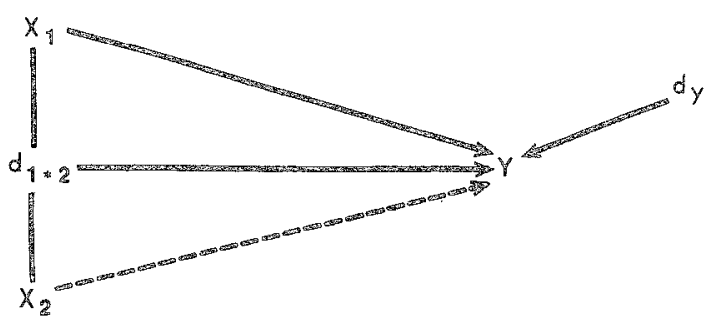

(b) Moderated Mediation

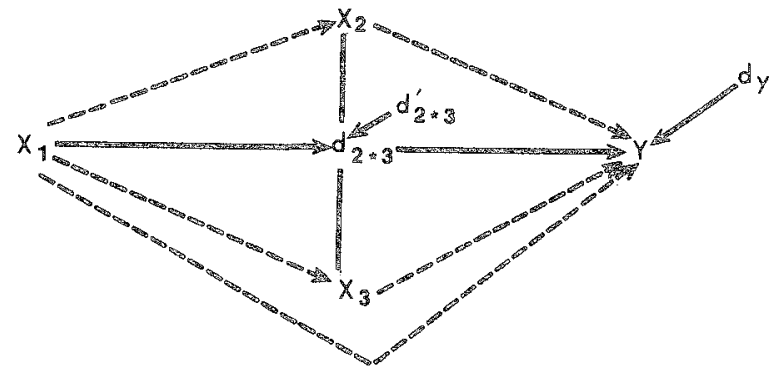

(c) Mediated Moderation

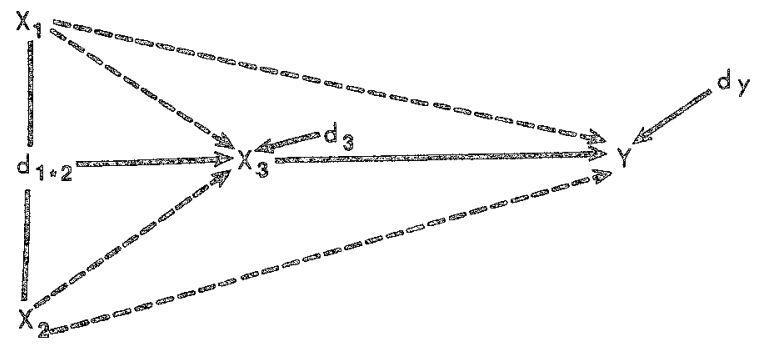

Baron and Kenny (1986) and James and Brett (1984) raised the possibility of moderated mediator effects and of mediated moderator effects. Two simple examples are shown in Figures $2 \mathrm{~b}$ and $2 \mathrm{c}$. Figure $2 \mathrm{~b}$ (moderated mediation) proposes a model in which the effect of an antecedent variable $X_{1}$ on a consequent variable $Y$ is transmitted through an interaction between two mediating variables $X_{2}$ and $X_{3}$ (solid lines). Broken lines indicate hypotheses of zero effects. The model in Figure $2 \mathrm{c}$ (mediated moderation) proposes that an interaction effect between two antecedent variables $X_{1}$ and $X_{2}$ is transmitted to the consequent variable $Y$ through a mediator $X_{3}$. Broken lines again indicate hypotheses of zero effects.

Models such as those in Figures $2 \mathrm{~b}$ and $2 \mathrm{c}$ may also be evaluated using the procedures described above. Significant effects predicted by Model $2 b$ are tested by regressing $Y$ on $d_{2 \times 3}$, and $d_{2 \times 3}$ on $X_{1}$, where, as in Equation 4,

$d_{2 \times 3}=X_{2 \times 3}-\hat{X}_{2 \times 3}=X_{2 \times 3}-\left(\hat{c}_{1} X_{2}+\hat{c}_{2} X_{3}\right)$, 
and $X_{2 \times 3}$ is the $X_{2} \times X_{3}$ cross-product. Significant regression parameter estimates would indicate support for model predictions. Zero effect hypotheses (broken lines) for the model in Figure $2 \mathrm{~b}$ may be evaluated by constructing the regression residual

$\tilde{d}_{Y}=Y-\hat{Y}=Y-\hat{b} d_{2 \times 3}$,

and estimating regression parameters (Lance, 1986):

$\tilde{d}_{Y}=f_{1} X_{1}+f_{2} X_{2}+f_{3} X_{3}+e_{Y}$

$X_{2}=f_{4} X_{1}+e_{2}$

$X_{3}=f_{5} X_{1}+e_{3}$,

where es again represent ots residuals. Nonsignificant estimates of the $f \mathrm{~S}$ in Equations 17 through 19 would support zero effect predictions. Significant estimates for one or more $f s$ would indicate specific misspecified zero effect hypotheses. Similarly, significant effects predicted by the model in Figure $2 \mathrm{c}$ are evaluated by regressing $Y$ on $X_{3}$, and $X_{3}$ on $d_{1 \times 2}$ (constructed as in Equation 4).

Disturbance-term regression tests of zero effect hypotheses (broken lines) proceed by constructing the residuals

$\tilde{d}_{Y}=Y-\hat{Y}=Y-\hat{b} X_{3}$

and

$\tilde{d}_{3}=X_{3}-\hat{X}_{3}=X_{3}-\hat{b} d_{1 \times 2}$

and estimating regression parameters in

$\tilde{d}_{Y}=f_{1} X_{1}+f_{2} X_{2}+f_{3} d_{1 \times 2}+e_{Y}$

and

$\tilde{d}_{3}=f_{4} X_{1}+f_{5} X_{2}+e_{3}$

Once again, nonsignificant estimates for $f \mathrm{~s}$ in Equations 22 and 23 would support model propositions, while significant $\hat{f}_{s}$ would indicate misspecified zero effect hypotheses.

\section{Exaruple Continued}

In the study described above, it was also hypothesized that Memory for Behaviors would have a direct effect on performance rating accuracy. Immediately after completing the memory test measure described above, respondents also rated the performance of the hypothetical lecturer on five 7-point dimensional performance rating scales. Dimensional rating accuracy was operationalized as the absolute deviation of each rating from the hypothetical lecturer's nominal performance dimension true score. For each respondent, overall Rating Accuracy was computed as the average of dimensional performance rating accuracy scores. ${ }^{3}$

All effects of Memory Demand and Cognitive Complexity on Rating Accuracy were presumed to be mediated by Memory for Behaviors. The path model depicting these hypotheses is shown in Figure 3. Nonzero effect hypotheses are shown as solid lines, zero effect hypotheses as broken lines.

Table 4 shows results of tests of predictions shown in the model in Figure 3 . Entries predicted to be nonzero are underlined; those predicted to be zero are nor underlined. Standardized regression estimates are shown.

${ }^{3}$ Note that this is actually a measure of rating inaccuracy. 
Figure 3

Path Model for Performance Rating Accuracy

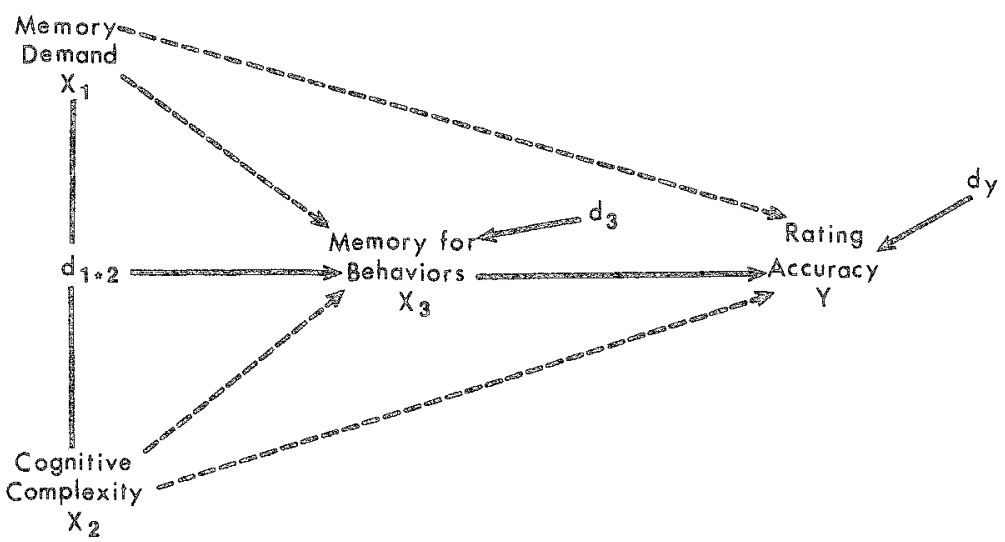

The first column in Table 4 reports regression results required to construct $d_{1 \times 2}$ residuals. The large $R^{2}=.953$ in this column points to the usual source of multicollinearity in HMR analysis, and corroborates results shown in Table $3 .^{4}$

The next two columns show oLs estimates for nonzero effects predicted by the model; both were significant. The last two columns show results from disturbance term regression tests of zero effect predictions (see Equations 20 and 21). As predicted, none were significant.

\section{General ERfect Analysis in Path Models Comtaining}

Direct, indirect, and total effects are easily calculated in models containing mediated moderation or moderated mediation effects. Procedures will be illustrated using the model in Figure $2 b$ (containing a mediating moderatory.

\begin{tabular}{|c|c|c|c|c|c|}
\hline \multirow[b]{2}{*}{ Predictor } & \multicolumn{5}{|c|}{ Dependent Variable } \\
\hline & $\mathrm{d}_{1 \times 2}$ & $Y$ & $x_{3}$ & $\tilde{d}_{\mathrm{Y}}$ & $\tilde{\mathrm{d}}_{3}$ \\
\hline $\mathrm{x}_{3}$ & - & $=.145 *$ & -- & -- & - \\
\hline$d_{1 \times 2}$ & $\cdots$ & $\cdots$ & $.163 *$ & -.078 & $-\infty$ \\
\hline $\mathrm{x}_{1}$ & $.867 * *$ & $=$ & $\cdots$ & -.086 & -.121 \\
\hline$x_{2}$ & $.628 * *$ & $\cdots$ & $\cdots$ & -.099 & -.039 \\
\hline $\mathrm{R}^{2}$ & $.953 * *$ & $.021 \%$ & $.027 \%$ & .020 & .014 \\
\hline
\end{tabular}

$* \mathrm{p}<.05 ; * x \mathrm{p}<.01$

This also represents the auxiliary regression equation recommended by Belsley et al. (1980) to detect the source(s) of collinearity indicated in the rop half of Table 3. 
The direct effects of variables are calculated from the structural equations by taking partial derivatives of the endogenous (dependent) variable with respect to each determinant (Stolzenberg, 1980). Sample estimates for parameters in structural equations for the model in Figure $2 b$ may be written:

$\hat{d}_{2 \times 3}=\hat{b}_{1} X_{1}$

$\hat{y}=\hat{b}_{2} d_{2 \times 3}$

Partial derivatives show the estimated direct effects of $d_{2 \times 3}$ on $Y$, and of $X_{1}$ on $d_{2 \times 3}$ :

$\partial d_{2 \times 3} / \partial X_{1}=\hat{b}_{1}$

$\partial Y / \partial d_{2 \times 3}=\hat{b}_{2}$

Effects of $X_{2}$ and $X_{3}$ on $Y$ are computed from

$\hat{Y}=\hat{b}_{2}\left(X_{2 \times 3}-\hat{c}_{1} X_{2}-\hat{c}_{2} X_{3}\right)$,

where $X_{2 \times 3}$ is the $X_{2} \times X_{3}$ cross-product, and are given as

$\partial Y / \partial X_{2}=\hat{b}_{2}\left(X_{3}-\hat{c}_{1}\right) \quad$,

$\partial Y / \partial X_{3}=\hat{b}_{2}\left(X_{2}-\hat{c}_{2}\right)$.

These results illustrate the symmetry of moderator effects.

Total effects are obtained from reduced-form equations. For the model in Figure $2 b$, these are

$\hat{d}_{2 \times 3}=\hat{b}_{1} X_{1}$

$\hat{Y}=\hat{b}_{2} d_{2 \times 3}=\hat{b}_{2}\left(X_{2 \times 3}-\hat{c}_{1} X_{2}-\hat{c}_{2} X_{3}\right)$,

and

$\hat{Y}=\hat{b}_{2} \hat{b}_{1} X_{1}$

from Equation 31. For the model in Figure 2b, the total effect of $X_{1}$ on $d_{2 \times 3}$ is calculated from Equation 31 and is simply $\partial d_{2 \times 3} / \partial X_{1}=\hat{b}_{1}$. Total effects of $X_{1}, X_{2}, X_{3}$, and $d_{2 \times 3}$ on $Y$ are

$\partial Y / \partial X_{1}=\hat{b}_{2} \hat{b}_{1}$,

$\partial Y / \partial X_{2}=\hat{b}_{2}\left(X_{3}-\hat{c}_{1}\right)$,

$\partial Y / \partial X_{3}=\hat{b}_{2}\left(X_{2}-\hat{c}_{2}\right)$,

and

$\partial Y / \partial d_{2 \times 3}=\hat{b}_{2}$.

Indirect effects are computed by subtracting direct effects from total effects. In the model in Figure $2 \mathrm{~b}$, the only indirect effect is from $X_{1}$ to $Y$ (i.e., $\hat{b}_{2} \hat{b}_{1}$ ).

The model in Figure 3 and results in Table 4 lend a more concrete example. Structural parameter estimates in the $X_{3}$ and $Y$ equations were

$\hat{X}_{3}=\hat{b}_{1} d_{1 \times 2}=.163 d_{1 \times 2}$

and

$\hat{Y}=\hat{b}_{2} X_{3}=-.145 X_{3}$

$\partial X_{3} / \partial d_{1 \times 2}=.163$ and $\partial Y / \partial X_{3}=-.145$ are the direct effects of $d_{1 \times 2}$ on $X_{3}$, and of $X_{3}$ on $Y$, respectively. Total effects are calculated from the reduced form equations:

$\hat{X}_{3}=\hat{b}_{1} d_{1 \times 2}=.163 d_{1 \times 2}$, 
$\hat{Y}=\hat{b}_{2} X_{3}=-.145 X_{3}$

and

$\hat{Y}=\hat{b}_{2} \hat{b}_{1} d_{1 \times 2}=-.145\left(.163 d_{1 \times 2}\right)$.

The total effects of $d_{1 \times 2}$ on $X_{3}\left(\partial X_{3} / \partial d_{1 \times 2}=.163\right)$ and of $X_{3}$ on $Y\left(\partial Y / \partial X_{3}=-.145\right)$ are equal to the direct effects. The total effect of $d_{1 \times 2}$ on $Y, \partial Y / \partial d_{1 \times 2}=(-.145)(.163)=-.024$, is an indirect effect.

\section{Discassion}

Pitfalls in interpreting individual regression parameter estimates are well known (see Ward, 1969). The residual-centered cross-product approach to regression tests of moderator effects, described above, is a method by which estimates of interaction effects are made more interpretable, symptoms of multicollinearity in HMR analysis are avoided, and hypothesis testing and effect analysis procedures can be performed in path models containing interactions.

The residual-centering approach was presented as an alternative to HMR analysis because of the current interest in testing person-situation interaction hypotheses (e.g., Bowers, 1973; Ekehammer, 1974; Epstein \& O'Brien, 1985) and moderator hypotheses in general (Baron \& Kenny, 1986). However, there are situations in which HMR may be preferable to the residual-centering approach. For example, if a researcher is only interested in comparing $R^{2} s$ to test for the presence of moderator effects, then HMR is simpler than the residual-centering approach while providing the same inferential statistics. HMR and residual-centering analyses will also yield identical results if predictor main effects are orthogonal to interactions (as in balanced ANOVA designs), and will yield equivalent results if they are nearly orthogonal. HMR may also be preferred in small sample sizes, as residual centering requires the estimation of two additional parameters (see Equation 4).

Residual centering will be an attractive alternative to HMR when (1) predictor main effects and the interaction are correlated and it is desired to interpret and test hypotheses about individual regression coefficients, (2) multicollinearity poses a threat to the integrity of HMR estimates, (3) sample sizes are moderate to large, or (4) decomposition of effects is desired (as in path analysis). The approach may also be viewed as a plausible alternative to other oLs applications, for example, polynomial regression tests of nonlinear effects. For instance, a presumed quadratic effect of some $X$ on $Y$ can be tested directly by constructing $d_{X^{2}}=X^{2}-\hat{X}^{2}=X^{2}-(\hat{b} X)$ and regressing $Y$ on $d_{X^{2}}$. Interpretational problems stemming from collinearity between $X$ and $X^{2}$ are thus avoided. Higher-order functions and cross-products among various powers of variables (Southwood, 1978; Stolzenberg, 1980) may be similarly treated.

For algebraic and interpretational simplicity, only standardized regression and effect analyses were discussed. In general, however, moderator hypotheses should be evaluated using unstandardized variables (Arnold, 1982). Heterogeneous variances or intercepts across subsamples may be disguised by standardized regression parameter estimates. Also, standard errors for standardized regression estimates may be miscalculated in the random predictor case (Bentler \& Lee, 1983). If these are concerns, unstandardized regression formulas may simply be substituted for standardized counterparts in this paper. Equations 6 through 8 demonstrate the ease of this substitution as well as the basic identity between reduced-form residual-centering and HMR analyses.

This paper shows that analysis of interaction effects in nonexperimental data need not be fraught with common interpretational problems. The residual-centering approach to testing interaction hypotheses in exploratory research is easily generalized to confirmatory analyses and decomposition of effects in path models. 


\section{References}

Arnold, H. J. (1982). Moderator variables: A clarification of conceptual, analytic, and psychometric issues. Organizational Behavior and Human Performance, 29, 143-174.

Arnold, H. I. (1984). Testing moderator variable hypotheses: A reply to Stone and Hollenbeck. Organizational Behavior and Human Performance, 34, $214-$ 224.

Baron, R. M., \& Kenny, D. A. (1986). The mediatormoderator variable distinction in social psychological research: Conceptual, strategic, and statistical considerations. Journal of Personality and Social Psychology, 51, 1173-1182.

Belsley, D. A. (1984). Collinearity and forecasting. Journal of Forecasting, 3, 183-196.

Belsley, D. A., Kuh, E., \& Welsch, R. E. (1980). Regression diagnostics: Identifying influential data and sources of collinearity. New York: Wiley.

Bentler, P. M., \& Lee, S.-Y. (1983). Covariance structures under polynomial constraints: Applications to correlation and alpha-type structural models. Journal of Educational Statistics, 8, 207-222.

Bentler, P. M., \& Woodward, J. A. (1979). Regression on linear composites: Statistical theory and applications. Multivariate Behavioral Research Monographs, No. 79-1.

Bowers, K. S. (1973). Situationism in psychology: An analysis and a critique. Psychological Review, 80 , 307-333.

Chatterjee, S., \& Price, B. (1977). Regression analysis by example. New York: Wiley.

Cohen, J. (1978). Partialed products are interactions; partialed powers are curve components. Psychological Bulletin, 85, 858-866.

Cohen, J., \& Cohen, P. (1975). Applied multiple regression/correlation analysis for the behavioral sciences. New York: Wiley.

Ekehammer, B. (1974). Interactionism in personality from a historical perspective. Psychological Bulletin, $81,1026-1048$

Epstein, S., \& O'Brien, E. J. (1985). The person-situation debate in historical and current perspective. $P_{S y}$ chological Bulletin, 98, 513-537.

Gulliksen, H., \& Wilks, S. S. (1950). Regression tests for several samples. Psychometrika, 15, 91-114.

James, L. R., \& Brett, J. M. (1984). Mediators, moderators and tests for mediation. Journal of Applied Psychology, 69, 307-321.

James, L. R., Mulaik, S. A., \& Brert, J. M. (1982). Causal analysis: Assumptions, models and data. Beverly Hills CA: Sage.

Johnston, J. (1984). Econometric methods. New York: McGraw-Hill.
Kerlinger, F. N., \& Pedhazur, E. J. (1973). Multiple regression in behavioral research. New York: Holt, Rinehart and Winston.

Lance, C. E. (1986). Disturbance term regression test procedures for recursive and nonrecursive models: Solution from intercorrelation matrices. Multivariate Behavioral Research, 21, 429-439.

McFatter, R. M. (1979). The use of structural equation models in interpreting regression equations including suppressor and enhancer variables. Applied Psychological Measurement, 3, 123-135.

Morris, J. H., Sherman, I. D., \& Mansfield, E. R. (1986). Failures to detect moderating effects with ordinary least squares-moderated multiple regression: Some reasons and a remedy. Psychological Bulletin, 99, 282-288.

Saunders, D. R. (1956). Moderator variables in prediction. Educational and Psychological Measurement, 16, 209-222.

Southwood, K. E. (1978). Substantive theory and statistical interaction: Five models. American Joumal of Sociology, 83, 1154-1203.

Stolzenberg, R. M. (1980). The measurement and decomposition of causal effects in nonlinear and nonadditive models. In K. F. Schuessler (Ed.), Sociological Methodology: 1980 (pp. 459-488). San Francisco: Jossey-Bass.

Stone, E. F., \& Hollenbeck, J. R. (1984). Some issues associated with the use of moderated regression. Or. ganizational Behavior and Human Performance, 34 , 195-213.

Tate, R. L. (1984). Limitations of centering for interactive models. Sociological Methods and Research, 13, 251-271.

Tzelgov, J., \& Henik, A. (1985). A definition of suppression situations for the general linear model: A regression weights approach. Educational and $P$ sychological Measurement, 45, 281-284.

Vannoy, 1. S. (1965). Generality of cognitive complexity-simplicity as a personality construct. Journal of Personality and Social Psychology, 2, 385-396.

Ward, $\mathbb{J} . \mathbb{H}$. (1969). Partitioning of variance and contribution or importance of a variable: A visit to a graduate seminar. American Educational Research Journal, 6, 467-474.

Zedeck, S. (1971). Problems with the use of moderator variables. Psychological Bulletin, 76, 295-310.

\section{Acknowledgriments}

The author thanks Bill Alley and Bill Graziano for motivating this effort, and John Cornwell, Dan Batson, the 
editor, and two anonymous reviewers for their helpful comments on an earlier draft of this paper.

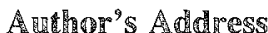

Send requests for reprints or further information to Charles E. Lance, Department of Psychology, University of Georgia, Athens GA 30602, U.S.A. requires payment of royalties through the Copyright Clearance Center, http://www.copyright.com/ 